\title{
Pharmacological rescue of adult hippocampal neurogenesis in a mouse model of X-linked intellectual disability
}

\author{
Manuela Allegra a,b,c,*, Cristina Spalletti a , Beatrice Vignoli d,e , Stefano Azzimondi ${ }^{\mathrm{a}}$, Irene Busti ${ }^{\mathrm{a}}$, Pierre Billuart ${ }^{\mathrm{f}}$, \\ Marco Canossa ${ }^{\mathrm{d}, \mathrm{e}}$, Matteo Caleo ${ }^{\mathrm{a}, * *}$ \\ a CNR Neuroscience Institute, via G. Moruzzi 1, 56124 Pisa, Italy \\ b Laboratorio Nest, Scuola Normale Superiore, P.zza San Silvestro 12, 56127 Pisa, Italy \\ c Accademia Nazionale dei Lincei, via della Lungara 10, 00165 Rome, Italy \\ d University of Trento, Centre for Integrative Biology (CIBIO), via Sommarive 9, 38123 Povo (TN), Italy \\ e European Brain Reasearch Institute (EBRI)-Rita Levi-Montalcini, via del Fosso di Fiorano 64, 00143 Rome, Italy \\ ${ }^{\mathrm{f}}$ Institute Cochin, INSERM U1016, Paris Descartes University, 24 rue du Fg St Jacques, 75014 Paris, France
}

\section{A R T I C L E I N F O}

\section{Article history:}

Received 12 September 2016

Revised 23 December 2016

Accepted 10 January 2017

Available online 12 January 2017

\section{Keywords:}

Rho GTPase

Axon extension

Dendritic spines

Fasudil

\begin{abstract}
A B S T R A C T
Oligophrenin-1 (OPHN1) is a Rho GTPase activating protein whose mutations cause X-linked intellectual disability (XLID). How loss of function of Ophn1 affects neuronal development is only partly understood. Here we have exploited adult hippocampal neurogenesis to dissect the steps of neuronal differentiation that are affected by Ophn1 deletion. We found that mice lacking Ophn1 display a reduction in the number of newborn neurons in the dentate gyrus. A significant fraction of the Ophn1-deficient newly generated neurons failed to extend an axon towards CA3, and showed an altered density of dendritic protrusions. Since Ophn1-deficient mice display overactivation of Rho-associated protein kinase (ROCK) and protein kinase A (PKA) signaling, we administered a clinically approved ROCK/PKA inhibitor (fasudil) to correct the neurogenesis defects. While administration of fasudil was not effective in rescuing axon formation, the same treatment completely restored spine density to control levels, and enhanced the long-term survival of adult-born neurons in mice lacking Ophn1. These results identify specific neurodevelopmental steps that are impacted by Ophn 1 deletion, and indicate that they may be at least partially corrected by pharmacological treatment.
\end{abstract}

(c) 2017 The Authors. Published by Elsevier Inc. This is an open access article under the CC BY-NC-ND license (http://creativecommons.org/licenses/by-nc-nd/4.0/).

\section{Introduction}

Intellectual Disability (ID) is a complex disease of the central nervous system (CNS) that results in impaired cognitive abilities (Chelly et al., 2006; Van Bokhoven, 2011). A genetic contribution to the etiology of ID is well established and, among the genetic conditions, the most frequent are the X-linked intellectual disabilities (XLIDs) forms caused by single gene mutations on the X chromosome (Humeau et al., 2009; Ropers and Hamel, 2005). Among the XLID genes, oligophrenin-1 (Ophn1) encodes a RhoGTPase-activating protein (Rho GAP) which is expressed in the developing and adult nervous system (Billuart et al., 1998; Fauchereau et al., 2003; Khelfaoui et al., 2007; Valnegri et al., 2011). At the cellular level, the protein is present both in glial cells and neurons where it colocalizes with F-actin, notably at the tip of growing dendrites (Fauchereau et al., 2003). OPHN1 is detected on

\footnotetext{
* Correspondence to: M. Allegra, CNR Neuroscience Institute, via G. Moruzzi 1, 56124 Pisa, Italy. Present address: Laboratory G5 Circuits Neuronaux, Institut Pasteur, 25 Rue du Dr Roux, 75724 Paris, France.

** Corresponding author.

E-mail addresses: manuela.allegra@in.cnr.it (M. Allegra), caleo@in.cnr.it (M. Caleo).

Available online on ScienceDirect (www.sciencedirect.com).
}

both sides of the synapse, indicating that it participates to synaptic function (Nadif Kasri et al., 2009; Nakano-Kobayashi et al., 2014; Khelfaoui et al., 2009; Nakano-Kobayashi et al., 2009). Indeed, decreased or defective Ophn1 signaling prevents synapse maturation and causes loss of synaptic structure, function and plasticity (Govek et al., 2004; Khelfaoui et al., 2007; Powell et al., 2012; Powell et al., 2014). Ophn1 knock-out (KO) mice, the murine model of the disease, exhibit behavioral impairments in spatial memory, and an immature phenotype of dendritic spines in hippocampal pyramidal neurons associated with altered synaptic plasticity (Khelfaoui et al., 2007; Khelfaoui et al., 2014). However, how Ophn1 loss of function impacts on neuronal development and circuit formation, leading to impaired information processing remains still incompletely understood. This is mainly due to the technical difficulties to study embryonic/perinatal neurogenic processes (i.e. proliferation, migration, morphological differentiation and functional integration) that largely overlap in time.

Here, we have examined how lack of Ophn1 impacts on neuronal maturation by following the process of adult neurogenesis - i.e. the morphological and functional development of new dentate granule neurons that are normally and constantly added to hippocampus (Kempermann et al., 2015; Braun and Jessberger, 2014; Christian et al., 2014). In 
mammals, new neurons are produced in two specific neurogenic niches, the subventricular zone (SVZ) and the subgranular zone (SGZ) in the dentate gyrus (DG) of the hippocampus. Adult hippocampal neurogenesis recapitulates at least some neuronal developmental processes including proliferation of progenitors, morphological differentiation and integration of newborn neurons into brain networks (Kempermann et al., 2015). In the SGZ, stem/progenitor cells proliferate and give rise to neuroblasts that localize within the granule cell layer of the DG, elaborate their dendritic arbor into the molecular layer and extend axons to innervate target cells in the CA3 region. Adult newborn neurons finally receive functional excitatory and inhibitory synaptic connections in order to be integrated in the existing hippocampal circuitry (Kempermann et al., 2015; Braun and Jessberger, 2014; Christian et al., 2014; Toni and Schinder, 2016). Principal dentate granule cells are the only neuronal subtype that is generated, and newly generated neurons have enhanced synaptic plasticity properties that enable them to contribute to specific forms of hippocampal-dependent learning and memory (Deng et al., 2010; Christian et al., 2014; Schmidt-Hieber et al., 2004). Accordingly, impairments in adult hippocampal neurogenesis have been suggested as one of the processes underlying learning and memory impairments in neurodevelopmental disorders (Contestabile et al., 2013; Guo et al., 2011; Ishihara et al., 2010; Giacomini et al., 2015; reviewed in Pons-Espinal et al., 2013a).

In this manuscript, we used Ophn1 KO mice to investigate how loss of Ophn1 affects adult neurogenesis in the DG. We observed deficits in newborn neuron survival, while proliferation of stem/progenitor cells was not affected. We also found an altered morphological maturation of newly generated cells with a robust impairment of axonal extension and immature dendritic spines in mice lacking Ophn1. Moreover, we also tested a rescue strategy based on inhibition of two kinases, Rho-associated protein kinase (ROCK) and protein kinase A (PKA), whose activity is potently up-regulated by loss of Ophn1 (Khelfaoui et al., 2014). In particular, we administered the ROCK/PKA inhibitor fasudil to Ophn1 KO mice, and found that dendritic spine alterations and survival of newborn neurons in the granule cell layer of DG could be restored upon this pharmacological treatment.

\section{Materials and methods}

\subsection{Animals and treatment}

All procedures conformed to the EU Directive 2010/63/EU for animal experiments and to the ARRIVE guidelines. Experimental protocols were approved by the Italian Ministry of Health. All analyses were performed blind to genotype and treatment. Experiments were carried out using male KO (Ophn-1-/y) and their control WT littermates (Ophn- $1^{+/}$ y) (Khelfaoui et al., 2007; Meziane et al., 2016). Mice were housed in a room with a temperature of $21^{\circ} \mathrm{C}, 12 \mathrm{~h}$ light/dark cycle, and food and water available ad libitum. All animals were $2-3$ months old at the beginning of the experiments. No mice were excluded from the analyses. Heterozygous females were bred with C57/Bl6 wild type mice to maintain the genetically modified colony. The animals were genotyped using the polymerase chain reaction (PCR) with a set of 2 oligonucleotides previously validated (Khelfaoui et al., 2007).

For the rescue experiments, $\mathrm{KO}$ and control littermate mice were treated with the clinically approved ROCK and PKA inhibitor fasudil for 7 weeks. At 2 months of age, mice were randomly assigned to the treatment or control groups. The animals in the treatment group received fasudil in the drinking water $(0.65 \mathrm{mg} / \mathrm{mL}$; Khelfaoui et al., 2014), while the controls received only water. The fasudil experiments always included a subset of naïve WT mice to be used for comparison.

\subsection{BrdU injections}

For evaluation of adult hippocampal neurogenesis in Ophn1 KO and WT mice, BrdU (Sigma-Aldrich) was administered intraperitoneally at
$50 \mathrm{mg} / \mathrm{kg}$ body weight. For the experiments on neuronal proliferation (Fig. 1A, C), mice received 2 injections spaced by $2 \mathrm{~h}$ and were sacrificed after $24 \mathrm{~h}$. For neuronal survival experiments, we performed $4 \mathrm{BrdU}$ injections every $2 \mathrm{~h}$ and animals were sacrificed at 15 or $50 \mathrm{dpi}$ (Fig. 1D, E; Kempermann et al., 2003). Because of the different doses of BrdU, and since independent cohorts of WT and KO mice were analyzed at each time point, the difference in BrdU-positive cells between genotypes was assessed by pairwise comparisons at 1, 15 and $50 \mathrm{dpi}$.

\subsection{Immunohistochemistry and stereological cell counting}

Animals were anesthetized with chloral hydrate and perfused through the heart with $4 \%$ paraformaldehyde (PFA) in $0.1 \mathrm{M}$ phosphate buffer (PB), pH 7.4. Brains were post fixed for $2 \mathrm{~h}$ at $4{ }^{\circ} \mathrm{C}$, and cryoprotected in $30 \%$ sucrose in $0.1 \mathrm{M} \mathrm{PB}$ at $4{ }^{\circ} \mathrm{C}$, and cut coronally at $40 \mu \mathrm{m}$ with a freezing microtome. For each animal, serial sections ( 1 out of 6) spanning the entire rostro-caudal extension of hippocampus were processed for different stainings. For BrdU detection, free-floating sections were pretreated by denaturing DNA with $2 \mathrm{M} \mathrm{HCl}$ for $30 \mathrm{~min}$ at $37^{\circ} \mathrm{C}$ (Rossi et al., 2006; Kempermann et al., 2003). Slices were blocked for $1 \mathrm{~h}$ at room temperature (RT) with $10 \%$ normal goat serum (NGS), $0.3 \%$ Triton $\mathrm{X}-100$ in PBS, and then incubated overnight at $4{ }^{\circ} \mathrm{C}$ with a rat monoclonal anti-BrdU antibody (1:200, Abcam, AB6326) diluted in NGS 5\% and Triton $0.1 \%$ in PBS. Signal was finally revealed with $2 \mathrm{~h}$ incubation at RT with Cy3 conjugated anti-rat IgG (1:500, Jackson Immuno Research). We also performed a double labeling for BrdU and neuronal nuclei (NeuN). Sections were incubated overnight with the rat antiBrdU antibody (1:200) and guinea pig polyclonal anti-NeuN (1:1000, Millipore, ABN90P). Bound primary antibodies were revealed with Cy3 anti-rat IgG (1:500) and AlexaFluor488 anti-guinea pig IgG (1:400, Jackson Immuno Research). For Dcx staining, sections were blocked for $1 \mathrm{~h}$ at RT with $10 \%$ rabbit serum (RS), $0.3 \%$ Triton X-100 in PBS, and then incubated overnight at $4{ }^{\circ} \mathrm{C}$ with goat polyclonal antiDcx primary antibody (1:1000, Santa Cruz, sc8066), $0.2 \%$ RS, and $0.1 \%$ Triton X-100 in PBS. The primary antibody was revealed by incubation for $2 \mathrm{~h}$ at RT with Rhodamine Red-X conjugated anti-goat IgG (1:500, Jackson Immuno Research) diluted in $1 \%$ RS and $0.1 \%$ Triton in PBS. To detect early neuronal cells expressing Dcx and NeuN, we performed a double staining for both. Sections were incubated with the goat anti$\operatorname{Dcx}(1: 1000)$ and guinea pig anti-NeuN $(1: 1000)$ overnight at $4{ }^{\circ} \mathrm{C}$. Signals were revealed with Rhodamine Red-X anti-goat IgG (1:500) and AlexaFluor488 anti-guinea pig IgG (1:400). All primary antibodies used in this report have been validated by several previous studies on hippocampal neurogenesis (Contestabile et al., 2013; Iggena et al., 2015; Vannini et al., 2016).

\subsubsection{Stereological counting}

The number of BrdU, Dcx and Dcx/NeuN positive cells was estimated in serial coronal sections covering the complete rostro-caudal extension of the DG as previously described (Rossi et al., 2006). Cells were counted on a fluorescence microscope (Zeiss) using a $20 \times$ objective and the analysis was performed using Stereo Investigator software (Microbrightfield). All cells in the granule cell layer and subgranular zone of every sixth section were counted, and the resulting number of cells was multiplied by six to give an estimate of the total number of labeled cells.

To investigate the fate of the newly generated cells, we performed the analysis of Dcx/NeuN and BrdU/NeuN double labeled neurons. For Dcx/NeuN staining, fluorescence images were captured with a microscope (Axio Imager. Z2, Zeiss) equipped with Apotome.2 (Zeiss) at resolutions of $1350 \times 1024$ pixels and a $63 \times$ EC-PLAN-NEOFLUAR oil objective ( $0.75 \mathrm{NA})$. For each experimental animal, 4 sections were analyzed, and for each section, z-stack images ( $1 \mu \mathrm{m}$ z-step size) of 3 different regions of the dorsal granule cell layer were acquired with the ZENpro software (Zeiss). For each animal, Dcx positive cells were analyzed for the colocalization with NeuN with the cell counter plug in 
A

BrdU injection

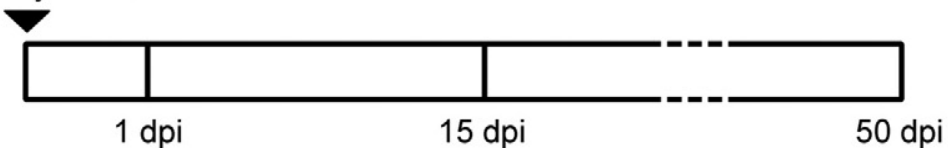

B
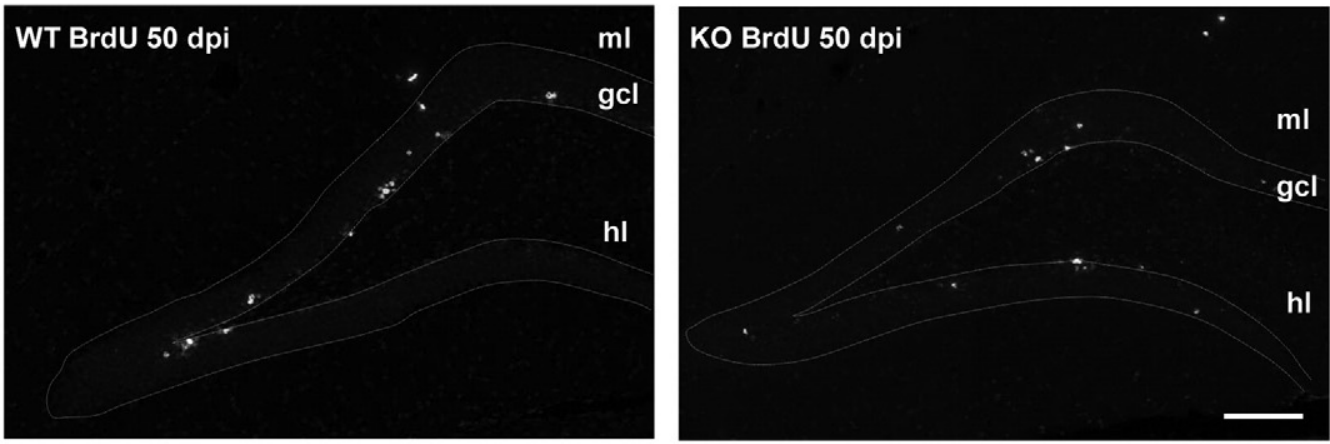

C $1 \mathrm{dpi}$

D

$15 \mathrm{dpi}$

E

$50 \mathrm{dpi}$

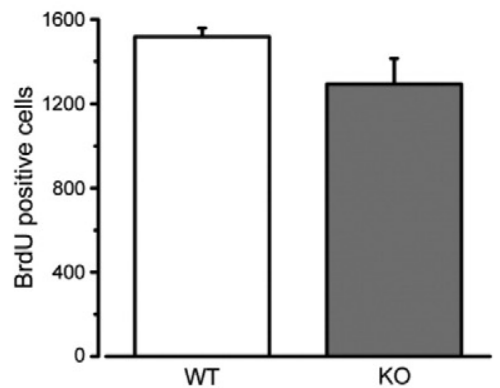

$\mathbf{F}$

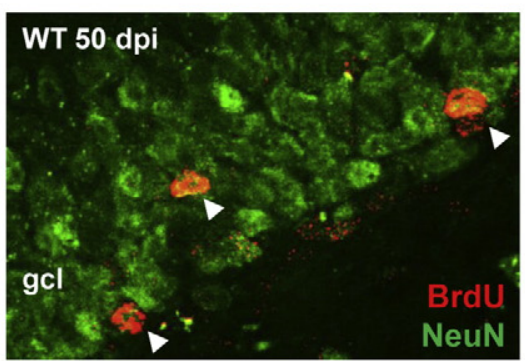

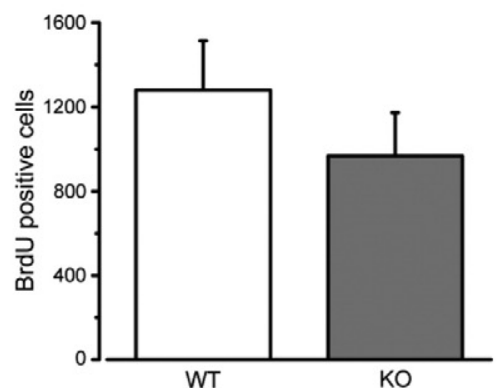

WT

$\mathrm{KO}$

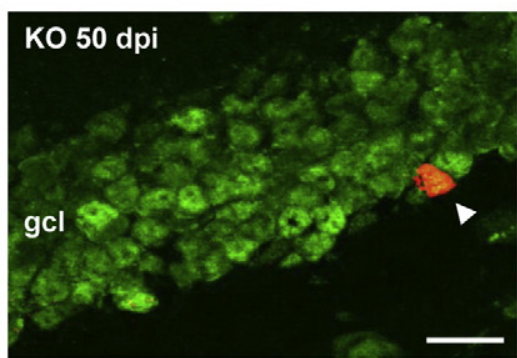

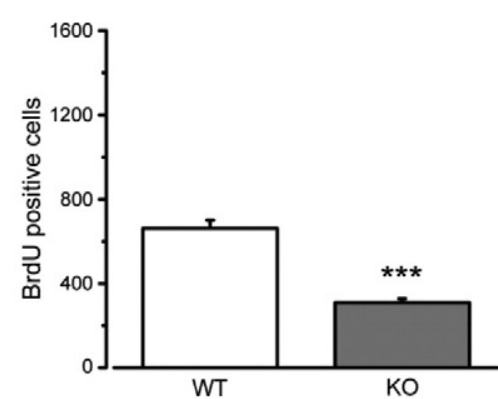

G

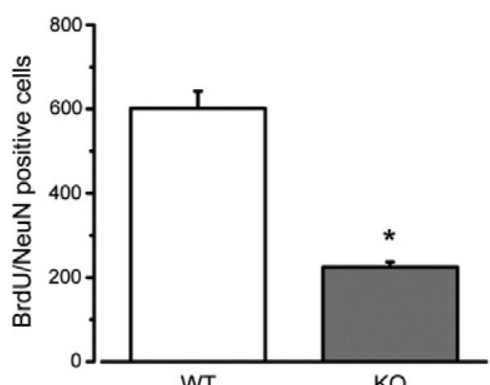

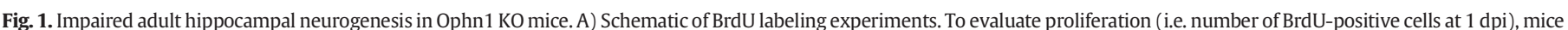

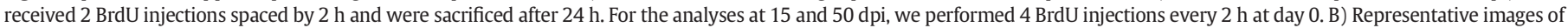

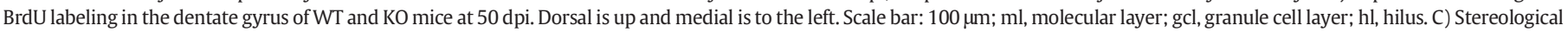

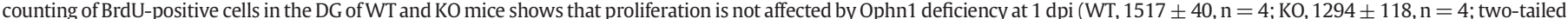

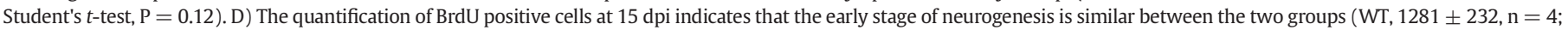

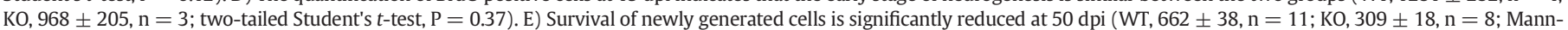

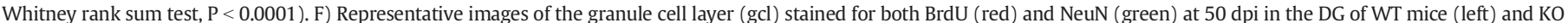

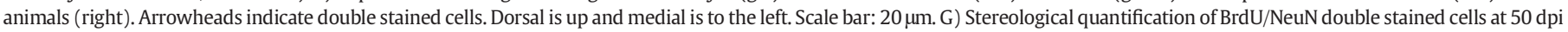

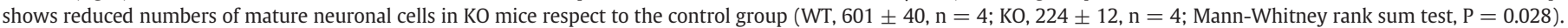
Histograms represent mean \pm SEM. Statistical significance, ${ }^{*} \mathrm{P}<0.05$; ${ }^{* * *} \mathrm{P}<0.001$.

(ImageJ) and in particular we evaluated the percentage of Dcx/NeuN early neurons over the total number of Dcx-positive cells. To evaluate BrdU/NeuN double staining, for each animal, $50 \mathrm{BrdU}$ positive cells were assessed for NeuN colocalization with a $40 \times$ objective and analysis was performed using Stereo Investigator software.

To quantify the effect of fasudil treatment on early neuronal cells, the numbers of Dcx-positive and Dcx-NeuN double labeled cells of each group (naïve $\mathrm{KO}$, KO treated with fasudil, WT treated with fasudil) were normalized to the mean of untreated WT mice of the same litter.
All experiments, data acquisition and quantification were performed blind to the genotype and treatment. The representative images shown in the Figures were obtained with a microscope (Axio Imager. Z2, Zeiss) equipped with Apotome.2 (Zeiss), using either a $20 \times$ EC-PLANNEOFLUAR objective or a $63 \times$ EC-PLAN-NEOFLUAR oil objective.

\subsubsection{Stereological volume analysis}

To determine the volume of the dentate gyrus and hilus, one-in-six series of sections was stained with Hoechst dye. In each section, the 
DG and hilus areas were traced at $10 \times$ magnification. The total volume was determined by summing the areas contoured in each section and multiplying the result by the distance between sections sampled $(300 \mu \mathrm{m})$

\subsection{Stereotaxic injections of retroviral vectors}

For virus delivery into dentate gyrus of the hippocampus, mice were anesthetized and a total volume of $1 \mu \mathrm{l}$ of retrovirus transducing murine Moloney leukemia virus (MoMulV)-based vector CAG-GFP (provided by Prof. F.H. Gage) was infused into each hemisphere (coordinates from Bregma: anteroposterior $-2 \mathrm{~mm}$, lateral $\pm 1.6 \mathrm{~mm}$, ventral $+1.8 \mathrm{~mm}$ ) through the insertion of capillary glasses (WPI) connected to a manual syringe pump (Narishige). Mice were allowed to recover and housed in standard cages until the day of sacrifice.

\subsection{Analysis of newborn DG granule cells morphology}

Confocal analysis was performed using a laser scanning motorized confocal system (Nikon $A 1$ ) equipped with an Eclipse Ti-E inverted microscope.

\subsubsection{Analysis of dendrites}

Coronal brain sections from injected brains ( $120 \mu \mathrm{m}$ thick) were acquired with a $20 \times$ oil objective and digital 2 magnification. Z-series stacks were acquired with an interval of $1 \mu \mathrm{m}$. Maximum intensity projections were produced using Image J software and tracing and quantification of dendrites were obtained using NeuronJ Plugin ((http:// imagescience.bigr.nl/meijering/software/neuronj). Only neurons showing intact dendritic arborization were analyzed. Sholl analysis was performed by Sholl Analysis Plugin (Ghosh Lab Website, http://biology. ucsd.edu/labs/ghosh/software/ShollAnalysis.class) using a $10 \mu \mathrm{m}$ interval between concentric circles.

\subsubsection{Analysis of spine density}

Spines were visualized using a $60 \times$ oil objective and digital 4 magnification. Z-series stacks were acquired with an interval of $0.3 \mu \mathrm{m}$. The area of analysis was set on dendritic segment located in the medial region of the molecular layer of the upper blade at approximately the same distance from the cell body. The number of spines was counted manually and density was calculated dividing the total number of spines by the length of dendritic segment. Three dimensional reconstruction was performed using Nis Element Software (Nikon).

\subsubsection{Analysis of axons}

To analyze axons of newborn neurons, cells were acquired with $60 \times$ oil objective with a z-series stack of $0.5 \mu \mathrm{m}$. Cells were classified for their orientation respect to the granular cell layer (horizontal or radial). Radially oriented cells were classified for presence of one single basal process extending to the hilus for a distance of at least $50 \mu \mathrm{m}$ (single axon) or for the presence of multiple short basal processes terminating within 20-30 $\mu \mathrm{m}$ from the cell body (no axon). Three dimensional (3D) reconstructions of newborn neurons were performed using Nis Element Software (Nikon) by using pseudocolor z-depth coding. By knowing the $3 \mathrm{D}$ coordinates of any point on the reconstruction we were able to determine the axon's profile including the axon's depth map, an image that contains information relating to the ending distance of an axon in respect to the cell body. To provide the distance information needed to establish axogenesis we used pseudo-colors: surfaces closer to the cell body are blue; surfaces further away from the cell body are red.

Mossy fibers labeled with GFP at CA3 region were quantified measuring mean fluorescence intensity in regions of interest (ROIs).

\subsection{Statistical analysis}

Statistical analysis was performed with SigmaPlot 12.0 software. Based on the preliminary analysis of statistical power, at least 3-6 mice were allocated to each experimental group. Pairwise comparisons of quantitative phenotypes between mice of different groups were assessed with a two-tailed Student's t-test when the observed treatment effects were normally distributed, or with the Mann-Whitney rank sum test when the samples were not drawn from normally distributed populations with the same variances. When more than two groups were analyzed, one way ANOVA followed by Holm-Sidak/Tukey/Dunn's post hoc test were used. The number of intersections in the Sholl analysis was examined with two-way repeated measures ANOVA, followed by Holm-Sidak test. Normality of distributions was assessed with Kolmogorov-Smirnov test. Level of significance was $\mathrm{P}<0.05$.

\section{Results}

\subsection{Ophn1 deletion impairs survival of newborn cells in the hippocampal dentate gyrus $(D G)$}

To investigate adult neurogenesis in Ophn $1^{-/ \mathrm{y}}$ mice, we performed experiments of bromo-deoxyuridine (BrdU) incorporation. In particular, we dated from birth (i.e. mitotic stage) the newly generated neurons by systemic injections of the thymidine analogue BrdU and assessed the total number of BrdU-positive cells 1, 15 and 50 days post-injection (dpi; Fig. 1A, B). This protocol allows visualizing proliferating cells and their progeny, i.e. those cells that incorporated the BrdU during the mitotic stage and then integrated in the DG circuitry.

The stereological analysis of adult hippocampal sections showed that, at 1 day after BrdU administration, the total number of newborn cells was similar in both groups, wild type (WT) and knock-out (KO) mice (Fig. 1C; Student's $t$-test, P>0.05). Next, we examined the survival of newly generated cells until 15 and $50 \mathrm{dpi}$. While at $15 \mathrm{dpi}$ there were no significant differences between the two groups (Fig. 1D; $t$-test, $\mathrm{P}>0.05$ ), we found a decrease of BrdU-positive cells at $50 \mathrm{dpi}$ in $\mathrm{KO}$ compared to WT littermates (Fig. 1B, E; Mann-Whitney test, $\mathrm{P}<0.0001$ ). Double labeling for BrdU and the neuronal marker NeuN was performed at 50 dpi to determine the phenotype of the BrdU-positive cells. We found a reduction of mature neuronal cells in $\mathrm{KO}$ mice with respect to the control group (Fig. 1F, G; Mann-Whitney rank sum test, $\mathrm{P}<0.05$ ), pointing to impaired addition of new neurons. These results indicate that loss of Ophn1 has no impact on cell proliferation while affecting the survival/integration of the newborn neurons in the DG hippocampal network.

Newly generated hippocampal cells committed to a neuronal fate begin expressing doublecortin (Dcx), a microtubule-associated protein that labels the newly generated neurons from the late precursor stage (about 4 days after birth) up to 4 weeks (Christie and Cameron, 2006; Fig. 2A). To quantify the total number of Dcx-positive cells, we performed the stereological counts in one hemisphere of brain sections of KO and WT animals and we found a substantial decrease of Dcx-positive newborn neurons in $\mathrm{KO}$ animals compared to controls (Fig. 2B, C; $t$-test, $\mathrm{P} \leq 0.001$ ). Between 21 and 28 days after birth, Dcx-positive newborn cells undergo a morphological and physiological development associated with the expression of neuronal nuclei (NeuN) upon final maturation (Fig. 2A). We performed a double staining with Dcx and NeuN (Fig. 2D) and we quantified the total number of early neuronal cells expressing both markers. The stereological results showed a significantly decreased number of total Dcx/NeuN positive cells in KO mice with respect to WT (Fig. 2E; t-test, $\mathrm{P}<0.001$ ).

Altogether, these data demonstrate that Ophn1 mainly affects the late stages of adult neurogenesis, when a postmitotic cell, already committed to the neuronal fate, is becoming a dentate granule cell.

Since previous studies have highlighted brain wide alterations in Opnh1 KO mice (Khelfaoui et al., 2007), we performed a 
A

Transition from

immature to mature

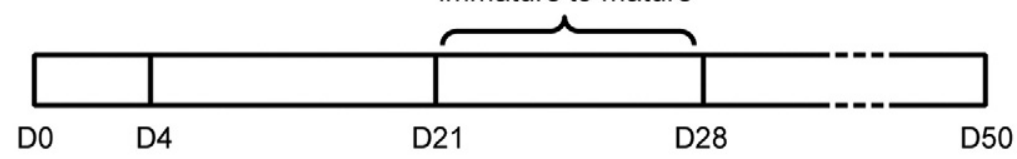

Dcx (immature neurons)

NeuN (mature neurons)

B
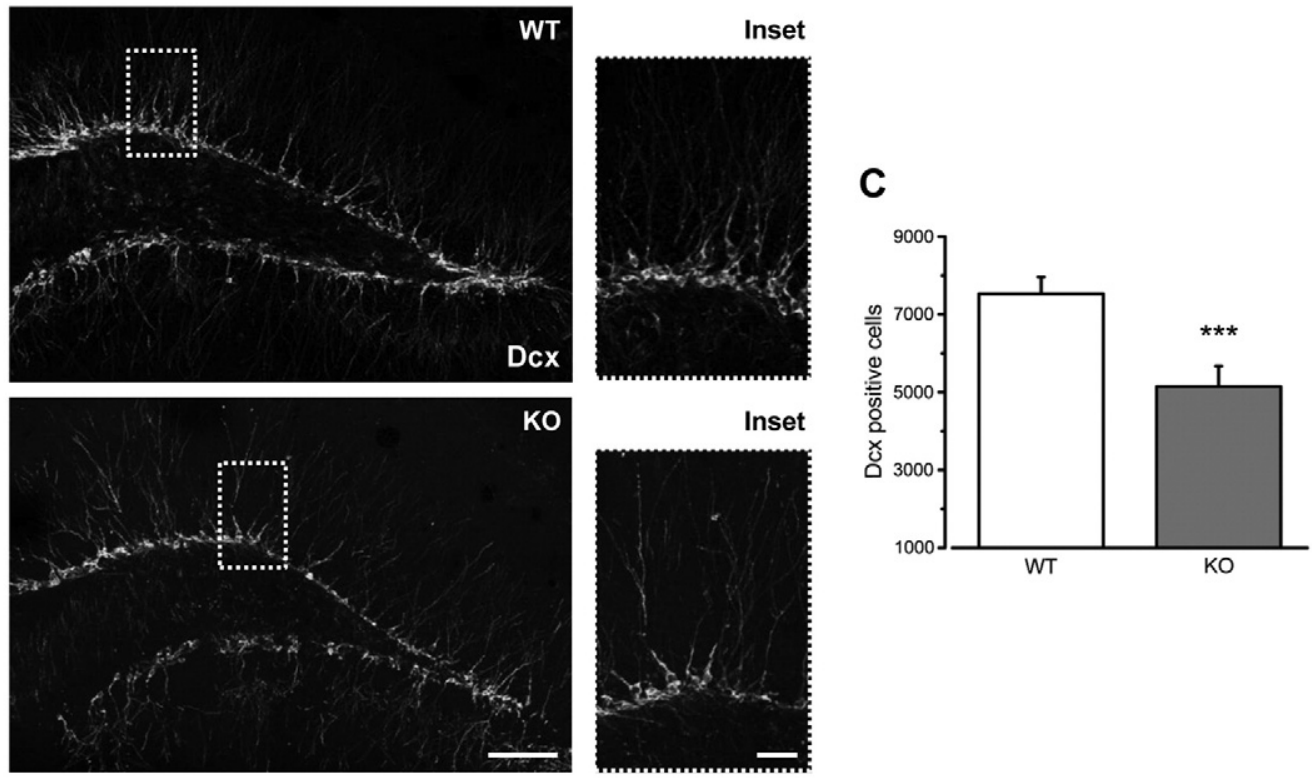

D
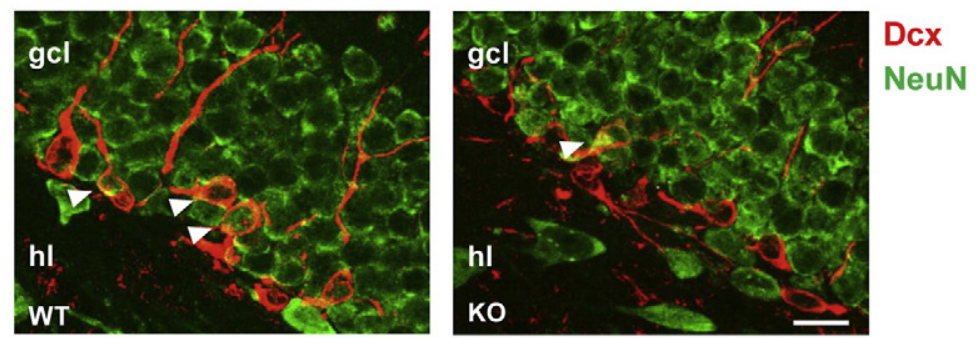

E

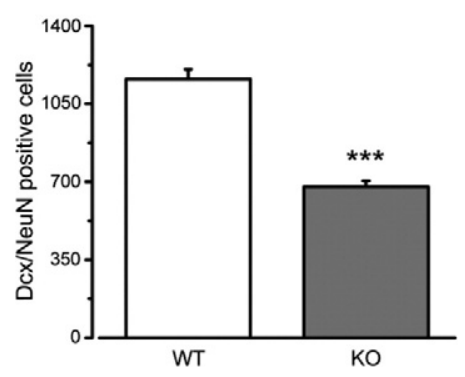

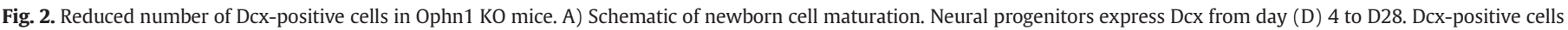

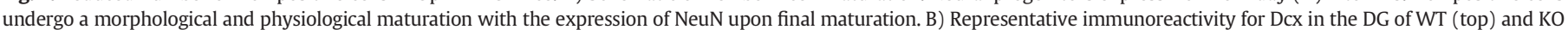

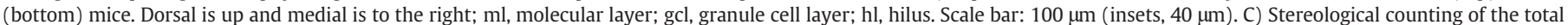

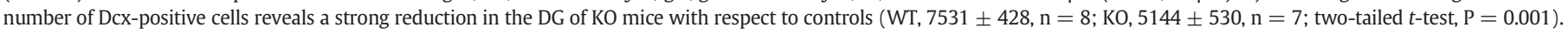

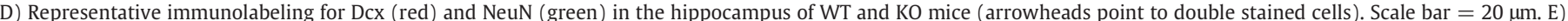

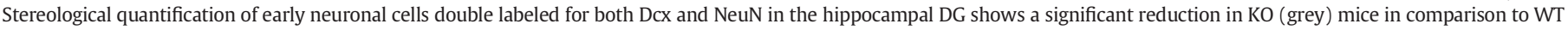
(white) animals (WT, $1161 \pm 44, \mathrm{n}=4 ; \mathrm{KO}, 678 \pm 26, \mathrm{n}=3$; two-tailed $t$-test, $\mathrm{P}=0.0003$ ). Histograms represent mean $\pm \mathrm{SEM}$. Statistical significance, ${ }^{* * *} \mathrm{P} \leq 0.001$.

neuroanatomical, stereological analysis of the volume of the neurogenic hippocampal niche. We found no differences in the volume of either granule cell layer or the hilus between WT and KO mice (t-test, $P>0.05$; Suppl. Fig. 1).

\subsection{Impaired morphological maturation of newborn neurons in Ophn1 KO} mice

The decrease of Dcx/NeuN double positive cells suggests an impairment of neuron integration in the pre-existing circuits of the dentate gyrus (DG) of Ophn1-deficient mice. Accordingly, we investigated axonal and dendritic maturation of newly generated neurons in Ophn1 KO mice and controls. Specifically, we injected WT and KO hippocampi with retroviral vectors expressing GFP to label proliferating cells (Bergami et al., 2008; Bergami et al., 2013; Zuccaro et al., 2014) and examined axonal formation at 21 and $28 \mathrm{dpi}$ (Fig. 3A). We found that while about $88 \%$ of the newborn cells in WT animals have extended an axon at 21 days, this percentage is dramatically reduced in $\mathrm{KO}$ mice (about 60\%; Fig. 3B-E; $t$-test, $P<0.05$ ). The majority of neurons from KO mice extended multiple small processes ending at a short distance from the cell body, as revealed by three-dimensional (3D) reconstruction images (Fig. 3C, D; right panels). Moreover, we quantified the presence of GFP-positive axons reaching the CA3 region by measuring fluorescence intensity in defined areas (Fig. 3B-D). In contrast to control mice, KO mice showed very little and thin axon fibers found in the CA3 region (Fig. 3C, D, F; t-test, $\mathrm{P}=0.032$ ). Impaired axogenesis was no 
A DAPI C

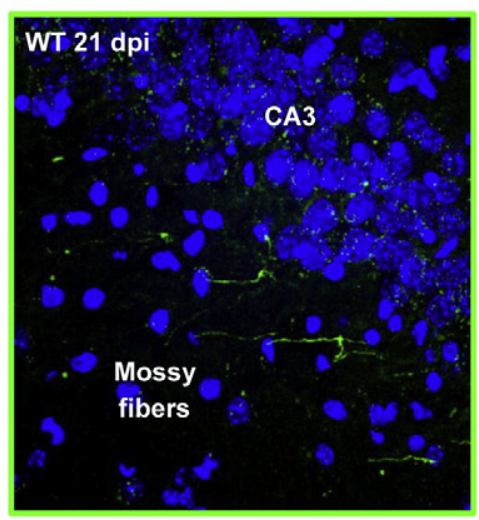

D

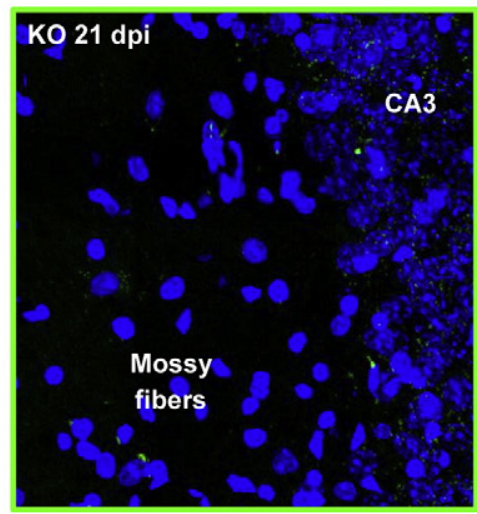

E
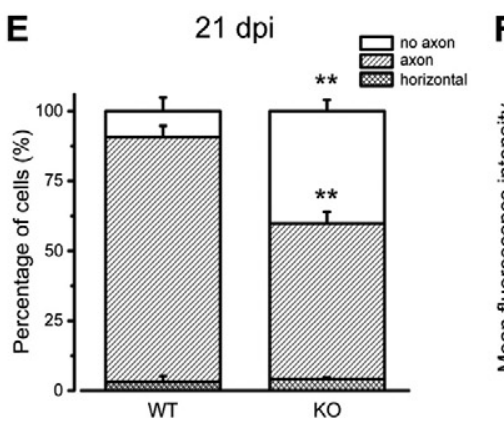

F
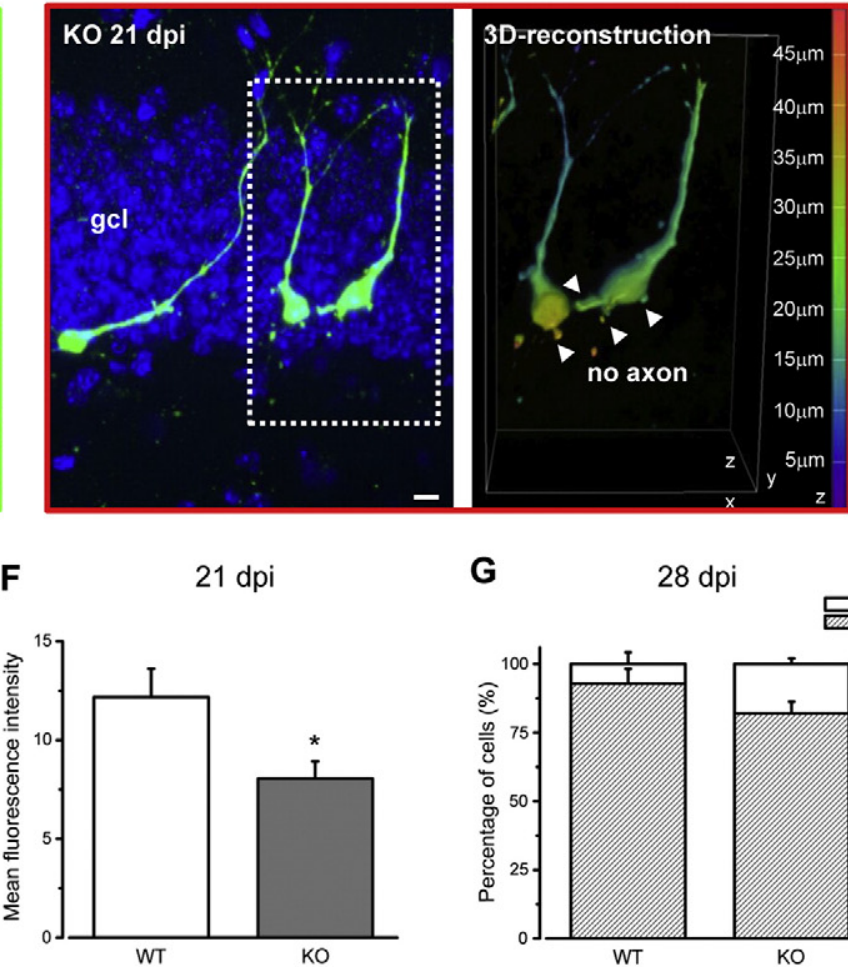

G
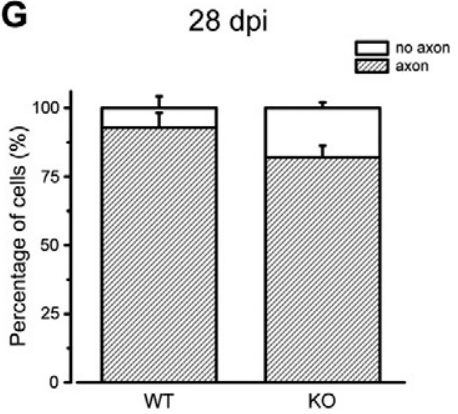
longer significant at $28 \mathrm{dpi}$ (Fig. 3G; $t$-test, $\mathrm{P}>0.05$ ). Thus, loss of Ophn1 impairs axon formation at a specific developmental stage in adult-generated neurons.

Prompted by the axonal phenotype, we reconstructed GFP-stained neurons in the DG to examine the dendritic structure of newborn cells at $21 \mathrm{dpi}$ (Fig. 4A). We found that overall dendritic length was not impacted by lack of Ophn1 (Fig. 4B; $t$-test, $\mathrm{P}>0.05$ ). Dendrite complexity was examined by Sholl analysis. We found a slight increase in the number of intersections between dendrites and Sholl circles at a distance of $180-230 \mu \mathrm{m}$ from the cell soma in the KO animals (two-way repeated measures ANOVA followed by Holm-Sidak test, $\mathrm{P}<0.0001$; Fig. $4 \mathrm{C}$ ).

We also examined density of spines on the dendrites of newborn DG neurons. We found that Ophn1-deficient mice displayed an overall greater density of dendritic protrusions at $21 \mathrm{dpi}$ (Fig. 4D, E; t-test, $\mathrm{P}<0.001$ ). In WT animals, density of dendritic spines was dramatically increased from 21 to $28 \mathrm{dpi}$, while this rate of increase was much less in $\mathrm{KO}$ mice (Fig. 4E; $t$-test, $\mathrm{P}<0.001$ ).

Overall, the morphological analysis reveals robust alterations in the structure of newborn neurons, in particular a reduction in the percentage of cells able to extend an axon towards CA3 and an alteration in the spine density in Ophn1-deficient mice.

\subsection{Rescue of adult hippocampal neurogenesis via systemic fasudil admin- istration in Ophn1 KO mice}

We next investigated whether the hyperactivation of the ROCK/PKA cascade, set in motion by Ophn1 deficiency (Govek et al., 2004; Khelfaoui et al., 2014), mediates the impairments of adult neurogenesis in Ophn $1^{-/ y}$ mice. We performed a rescue experiment by treatment with the clinically approved ROCK/PKA inhibitor fasudil (Khelfaoui et al., 2014; Meziane et al., 2016) and assessed whether this pharmacological strategy was able to restore the impairments in hippocampal neurogenesis.

As shown in Fig. 5A, B, seven weeks of fasudil administration were able to completely normalize the total number of Dcx-stained cells ( $t$ test, $\mathrm{P}<0.01$ ). The percentage of Dcx/NeuN double stained cells over the total sample of Dcx-positive cells was also rescued by fasudil treatment (Fig. 5C; $t$-test, $\mathrm{P}<0.05$ ).

We next evaluated the impact of fasudil delivery on the morphology of adult-generated neurons, 21 days after birth (21 dpi; Fig. 6A). The axonal phenotype was not impacted by fasudil and indeed we detected an abnormally low proportion (about $60 \%$ ) of granule cells with an axon extending towards CA3. This proportion was not different in fasudiltreated and untreated $\mathrm{KO}$ mice ( $t$-test, $\mathrm{P}>0.05$; Fig. 6B). In contrast, the spine alterations were rescued by fasudil treatment, with a significant decrease of the density of dendritic protrusions ( $t$-test, $\mathrm{P}<0.05$; Fig. 6C).

To ascertain whether fasudil treatment could normalize the number of mature newborn neurons, we evaluated the total number of BrdUpositive cells in the hippocampal DG, 50 days after injection. The data demonstrated that systemic fasudil administration partially rescues the impairment found at $50 \mathrm{dpi}$ (Fig. 6D, E). Fasudil-treated KO mice displayed a higher number of BrdU-positive cells in comparison to the $\mathrm{KO}$ vehicle animals (Mann-Whitney test, $\mathrm{P}<0.05$ ). Moreover, the quantification of BrdU and NeuN double stained cells demonstrated that fasudil treatment is able to increase the number of mature newborn neurons at 50 dpi in $\mathrm{KO}$ mice (Mann-Whitney rank sum test, $\mathrm{P} \leq 0.01$; Fig. $6 \mathrm{~F}, \mathrm{G})$. Overall, these data demonstrate that fasudil treatment for 7 weeks rescues a significant proportion of adult-generated neurons in Opnh1 KO mice.

As a control, we also checked whether fasudil treatment per se increases the neurogenic cellular population in the hippocampal DG of WT animals. As shown in Fig. 7, control and fasudil-treated WT mice displayed similar numbers of both Dcx-positive cells (Fig. 7A t-test, $\mathrm{P}>0.05$ ) and BrdU-labeled mature newborn neurons at $50 \mathrm{dpi}$ (Fig. $7 \mathrm{~B}, t$-test, $\mathrm{P}>0.05$ ). Thus, fasudil appears to restore impaired generation of newborn cells in Ophn1-deficient mice but has no impact on basal neurogenesis in healthy mice.

\section{Discussion}

Ophn1-dependent intellectual disability is a complex neurodevelopmental disorder involving several pathophysiological processes occurring in various brain areas. Studying adult hippocampal neurogenesis may be useful to shed light on the alterations in neuronal maturation that underlie cognitive impairment in XLID. Indeed, adult neurogenesis recapitulates at least some of the aspects of brain development and comprises a series of sequential events including proliferation of stem/progenitor cells, morphological maturation and functional integration of newly generated neurons into hippocampal circuitry. These steps have been characterized in detail (Kempermann et al., 2015; Braun and Jessberger, 2014; Christian et al., 2014; Bergami et al., 2015) and allow a window on brain development and hippocampal function (Toni and Schinder, 2016; Deng et al., 2010; Akers et al., 2014). Thus, adult neurogenesis represents an accessible model to dissect abnormalities in neuronal maturation/wiring, and test novel treatment options (Contestabile et al., 2013; Guo et al., 2011; Pons-Espinal et al., 2013b; Giacomini et al., 2015). Importantly, hippocampal neurogenesis deficits may contribute to the pathophysiology of intellectual disabilities, such as Rett, Fragile X and Down syndrome (Guo et al., 2011; Christian et al., 2014; Pons-Espinal et al., 2013a). The data presented here demonstrate that Ophn1 loss of function causes robust alterations in adult hippocampal neurogenesis, and suggest a possible therapeutic strategy for counteracting the impairments in neuronal maturation by inhibiting ROCK/PKA signaling pathways.

\subsection{Impaired adult hippocampal neurogenesis in mice lacking Ophn1}

The number of BrdU-labeled cells at 1dpi was superimposable in Ophn- $1^{+/ y}$ and Ophn- $1^{-/ y}$ mice, indicating that proliferation of stem/ progenitor cells was not impacted by Ophn1 deficiency. Similar data were obtained at 15 days. Analysis of Dcx-positive immature neurons, however, revealed a significant deficit in Ophn1 KO animals. Dcx expression extends from 4 days post birth up to a phase of postmitotic maturation lasting until about P28 (Christie and Cameron, 2006; Kempermann et al., 2015). Taken together, these data indicate that newly generated cells become particularly vulnerable to Ophn1 deficiency in a phase comprised between the third and fourth week post birth. Newborn neurons at 21 days also displayed significant alterations in neurite extension, with reduced axon formation, a phenotype that

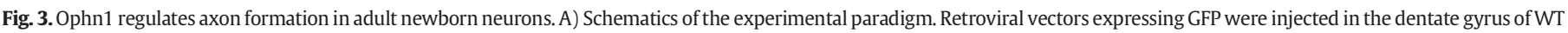

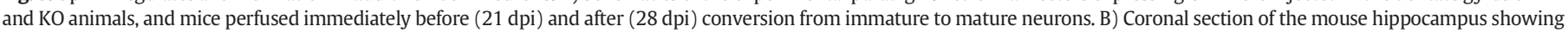

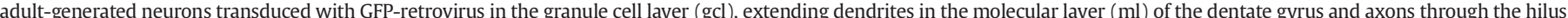

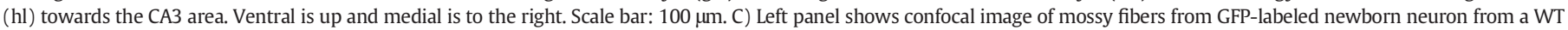

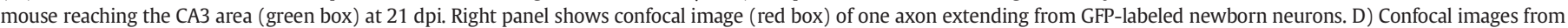

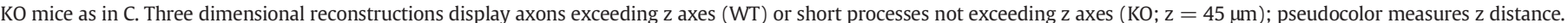

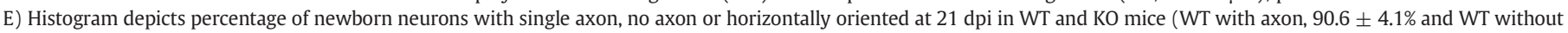

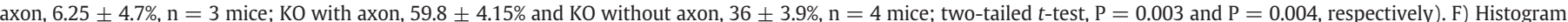

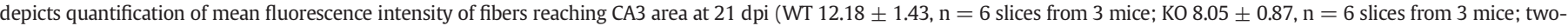

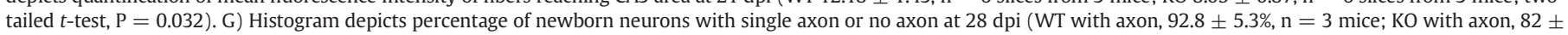
$4.1 \%, \mathrm{n}=4$ mice; two-tailed $t$-test, $\mathrm{P}=0.16$ ). Histograms represent mean $\pm \mathrm{SEM}$. Statistical significance, ${ }^{*} \mathrm{P}<0.05,{ }^{* *} \mathrm{P}<0.01$. 
A
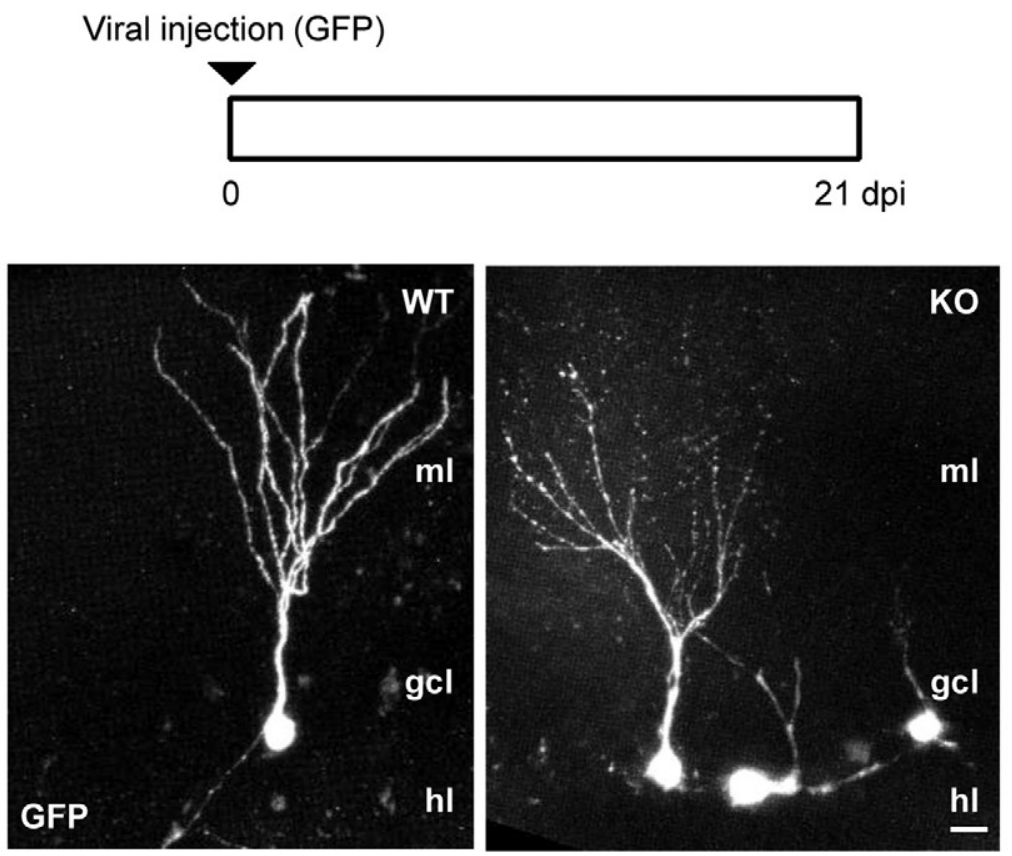

B

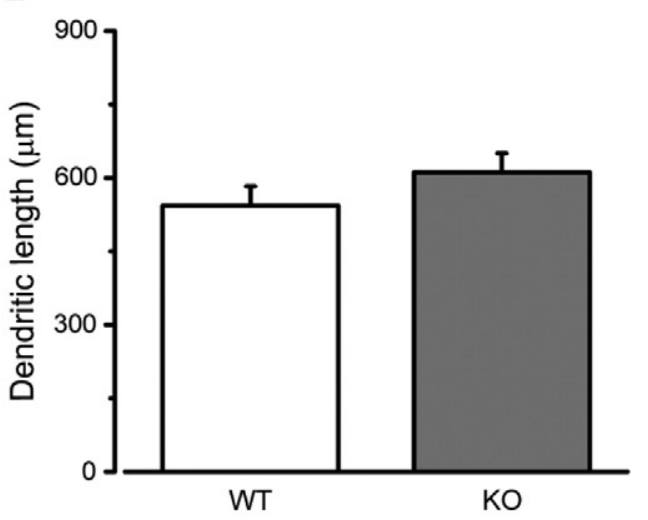

C

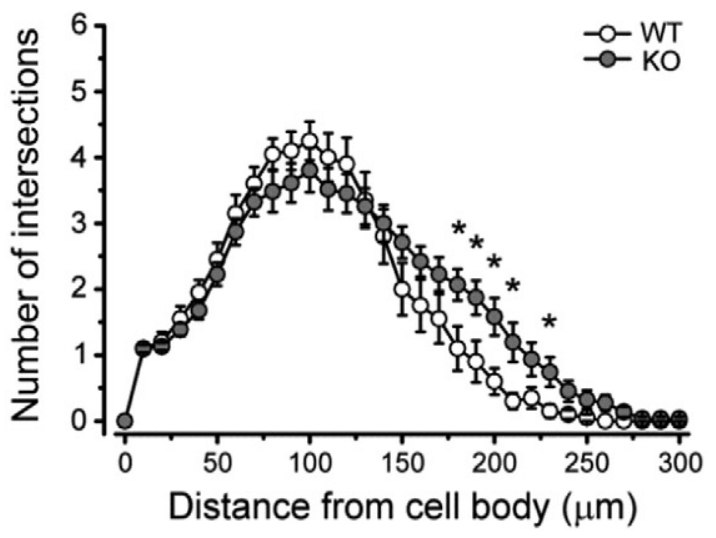

D

E
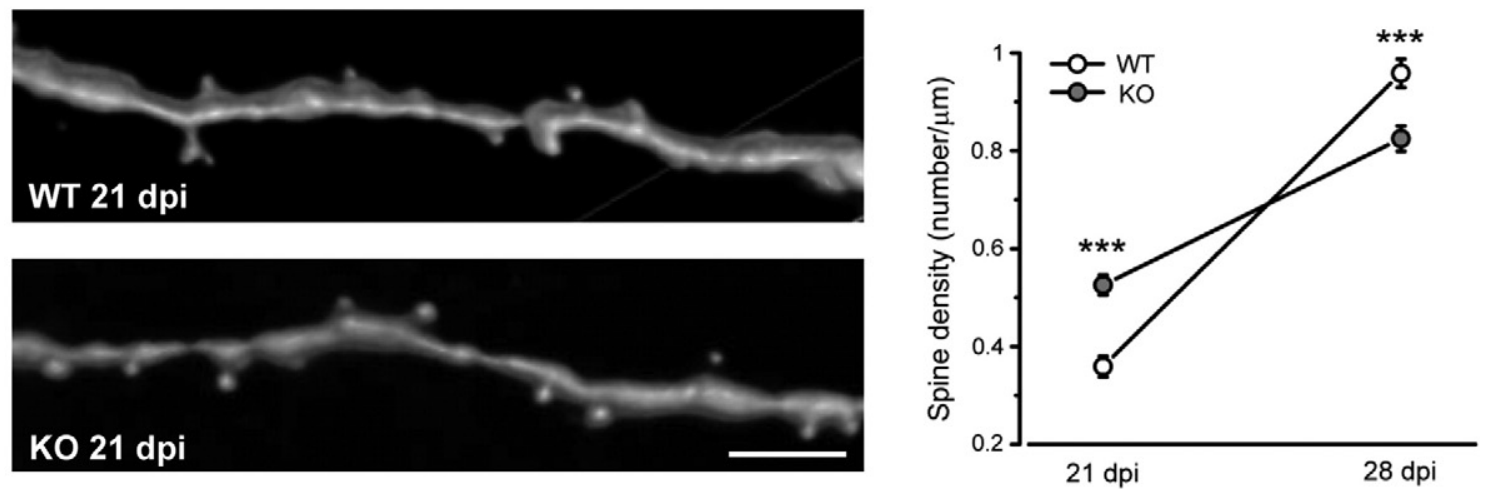

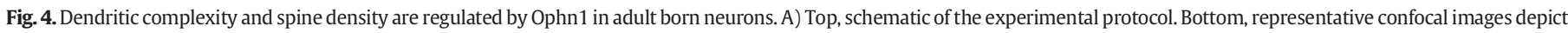

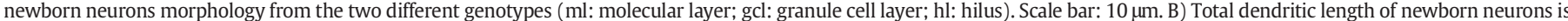

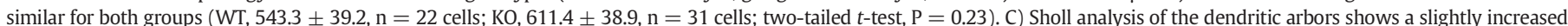

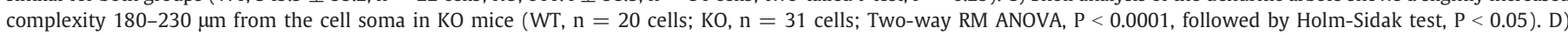

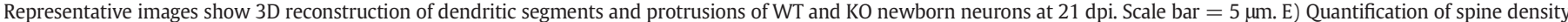

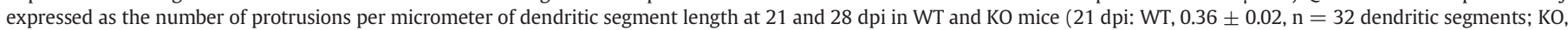

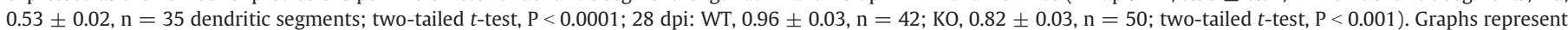
mean \pm SEM. Statistical significance, ${ }^{*} \mathrm{P}<0.05,{ }^{* * *} \mathrm{P} \leq 0.001$. 
A
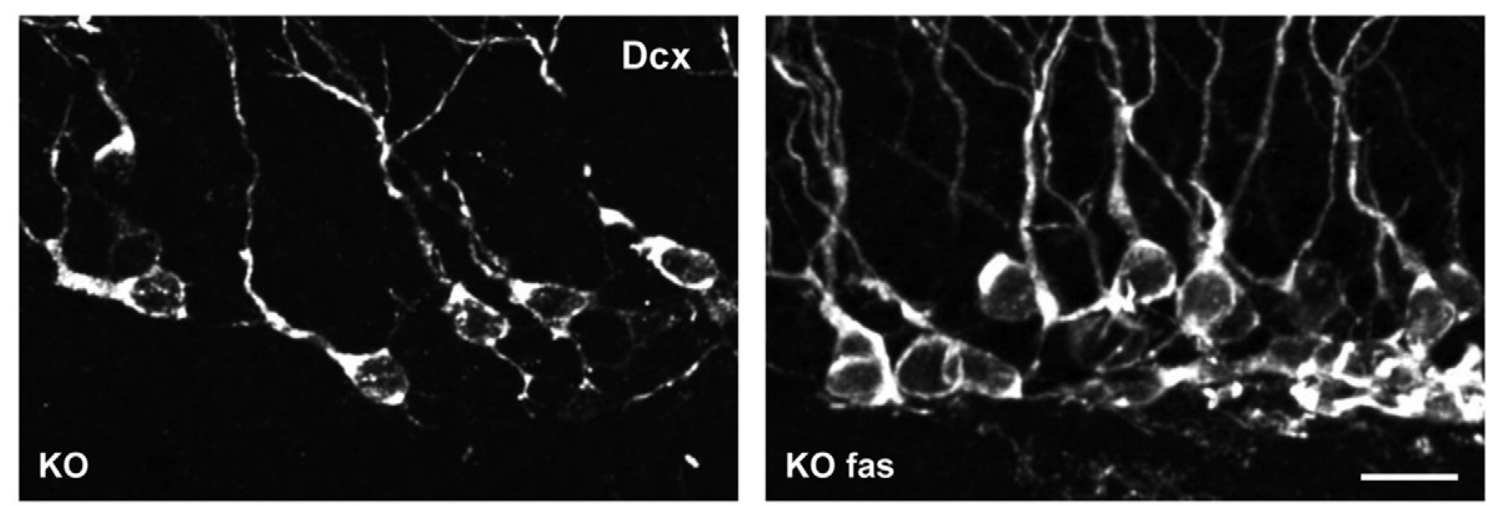

B
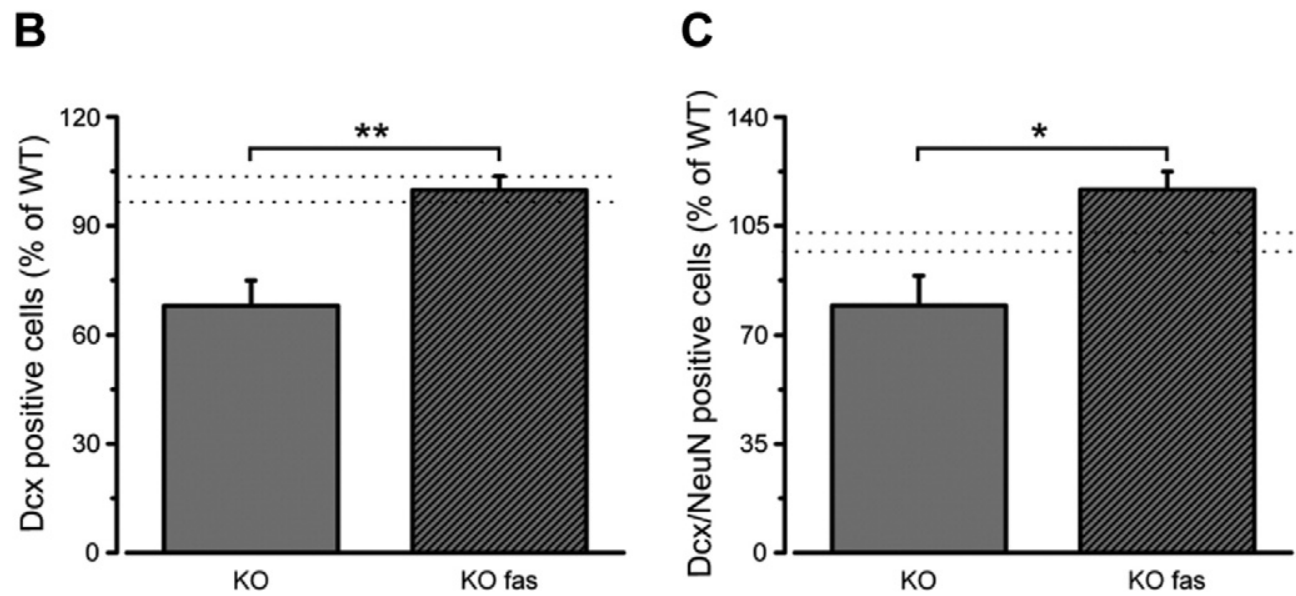

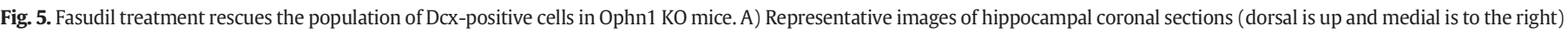

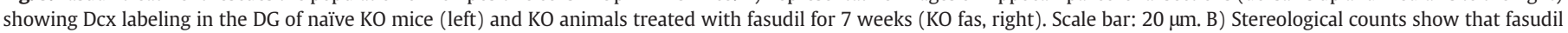

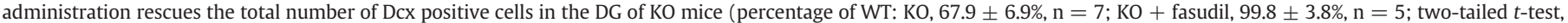

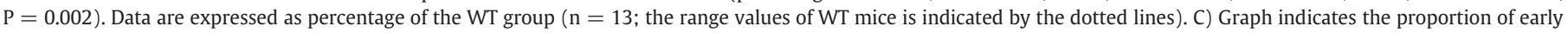

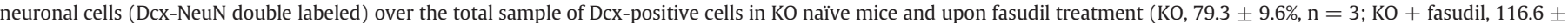

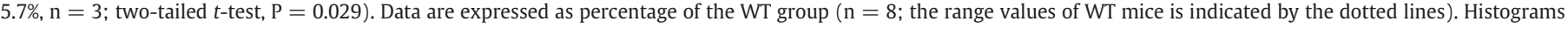
represent mean \pm SEM. Statistical significance, ${ }^{*} \mathrm{P}<0.05$, ${ }^{* *} \mathrm{P}<0.01$.

was not observed at 28 days (see Fig. 3 ). One possible explanation for this finding is a delay in axonal growth of neurons lacking Ophn1. It is also possible that Ophn1-deficient neurons unable to extend their axons towards CA3 undergo programmed cell death and are removed from the hippocampal network at the time of their integration. This is consistent with the robust drop of early neuronal cells (double labeled for Dcx and NeuN, corresponding to the time window 21-28 days post-birth) in the DG of Ophn1-deficient mice.

Maturation of newborn cells continues over the subsequent weeks and there is consensus in the literature that after a period of $7-$ 8 weeks, the new neurons become indistinguishable from the resident granule cells (Kempermann et al., 2015; Toni and Schinder, 2016). We therefore assessed the number of mature, BrdU-labeled neurons at $50 \mathrm{dpi}$. We found a significant decline in the number of newborn cells in the DG of Ophn1 KO mice. Thus, decreased new neuron production and integration may contribute to dysfunction of the hippocampus in Ophn1-dependent XLID (Khelfaoui et al., 2007; Meziane et al., 2016). Specifically, recent data indicate that high levels of adult hippocampal neurogenesis contribute to removal of context fear memory, in particular removal of context fear memory (Akers et al., 2014; Saxe et al., 2006). Consistent with these data, Ophn- $1^{-/ y}$ mice display a deficit in fear extinction memory (Khelfaoui et al., 2014).
4.2. Morphological abnormalities of newly generated granule cells in the DG of Ophn1 KO mice

To study the morphological maturation of adult-generated neurons we injected a retroviral vector expressing GFP into the DG (Zuccaro et al., 2014). We analyzed axon formation and dendritic structure by confocal microscopy. In the control mice, the vast majority (almost 90\%) of 21-days-old neurons displayed one axon traveling through the hilus and reaching the CA3 region. Conversely, loss of Ophn1 caused a significant decrease in the proportion of neurons forming an axon. This was demonstrated by both the 3D analysis of axonal formation and by the assessment of fluorescence intensity in CA3 region. However, it is difficult to establish whether these axon terminals form functional synaptic boutons onto the target pyramidal neurons. A role for OPHN1 in axon extension is supported by its localization at the tip of growing neurites (Fauchereau et al., 2003). To our knowledge, this is the first report of aberrant axon formation in Ophn1-dependent XLID. It is likely that alterations in long-range axonal extension may result in abnormal network connectivity and consequent deficits in information processing in XLID. Indeed disruptions in functional connectivity are a typical hallmark of neurodevelopmental disorders (Van Der Molen et al., 2014; Plitt et al., 2015). 
A

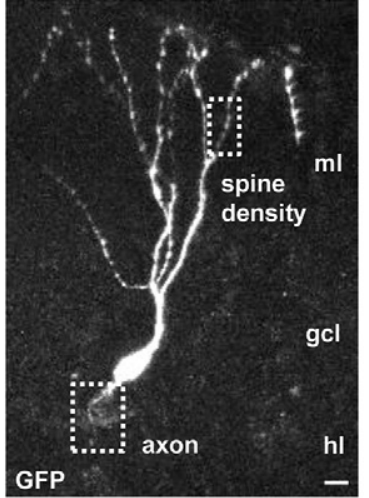

D

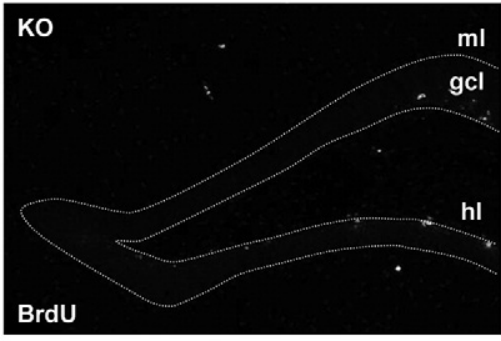

$\mathbf{F}$

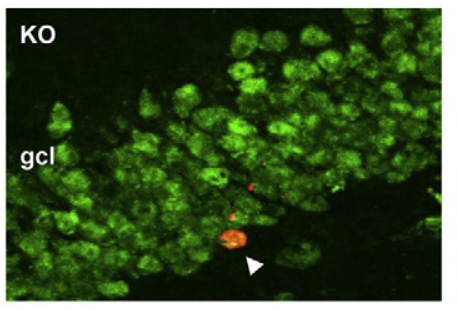

B

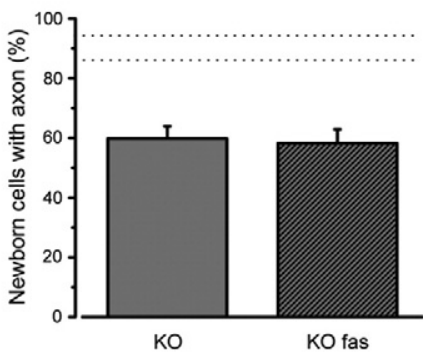

KO
Axonal phenotype
C

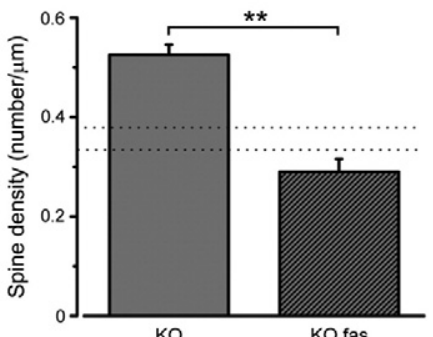

KO

Spine phenotype
E
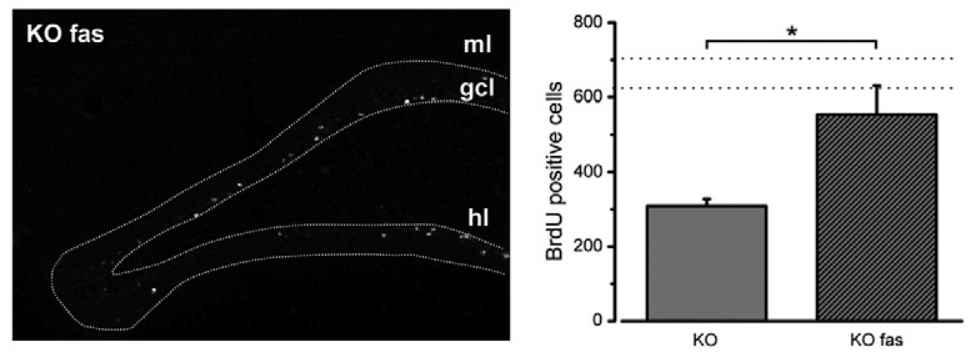

G

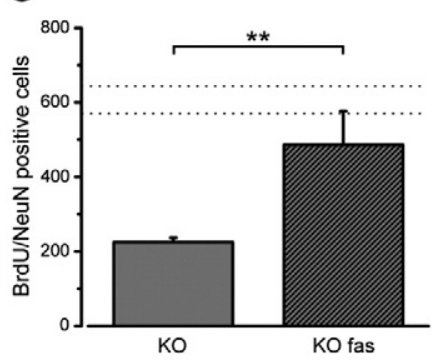

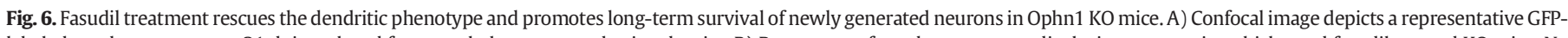

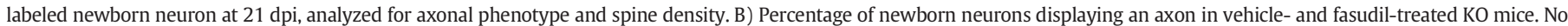

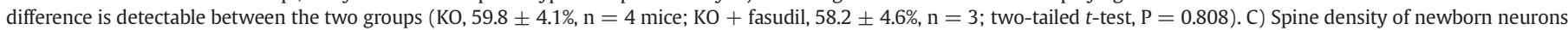

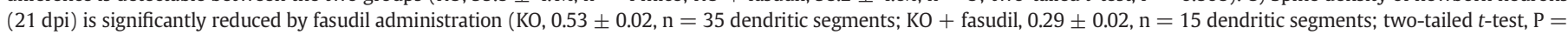

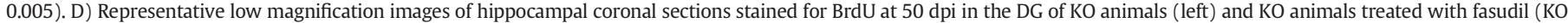

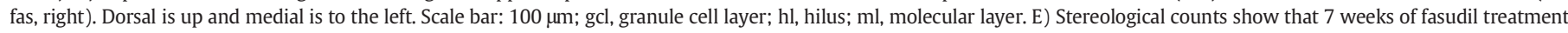

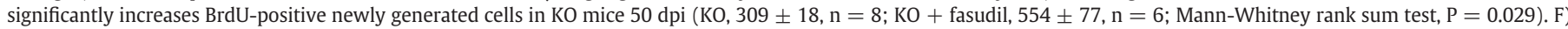

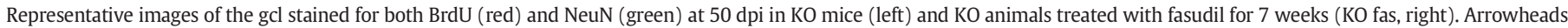

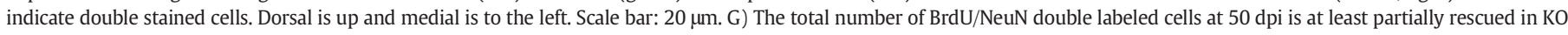

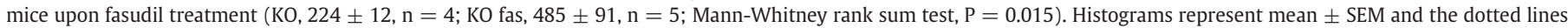
indicate the range values of the WT group. Statistical significance, ${ }^{*} \mathrm{P} \leq 0.05,{ }^{*} \mathrm{P} \leq 0.01$.

We also examined the structure of the dendritic arbor. Newly generated neurons start to extend dendritic processes into the molecular layer at around 7 days after birth (Zhao et al., 2006), and to form afferent connections with the local neuronal network during the third week after birth (Deng et al., 2010). In particular, adult-born neurons begin to elaborate spines that form synapses with the afferent perforant fibers from the entorhinal cortex, and this phase of synaptic integration is characterized by increased synaptic plasticity (Ge et al., 2007; Ge et al., 2008). We found that while total dendritic length was unaltered, Ophn1 deficiency produced an enhancement of the density of spines $21 \mathrm{dpi}$. At this stage of maturation, most of the dendritic protrusions had an immature appearance. Interestingly, the overall density of dendritic spines increases more robustly from 21 to $28 \mathrm{dpi}$ in WT than in $\mathrm{KO}$ animals. One possible explanation is that newly generated Ophn1 deficient neurons display an initial overproduction of immature spines that is later followed by a reduced addition, ultimately resulting in a lower density of mature protrusions. These data are consistent with previous findings reporting a key role for Ophn1 in dendritic spine morphogenesis. Indeed, inactivation of Ophn1 function increases the density and proportion of immature spines in neuronal cultures and in vivo (Khelfaoui et al., 2007; Govek et al., 2004; Redolfi et al., 2016). In the Ophn1 KO mouse, mature dendritic spines are reduced and this is associated with impairments of spatial learning and alterations of social behavior (Khelfaoui et al., 2007).

\subsection{Restoration of adult hippocampal neurogenesis by inhibition of ROCK/ PKA signaling}

One of the best known downstream target of OPHN1 is the Rho kinase (ROCK) protein. Ophn1 loss of function results in persistent 
A

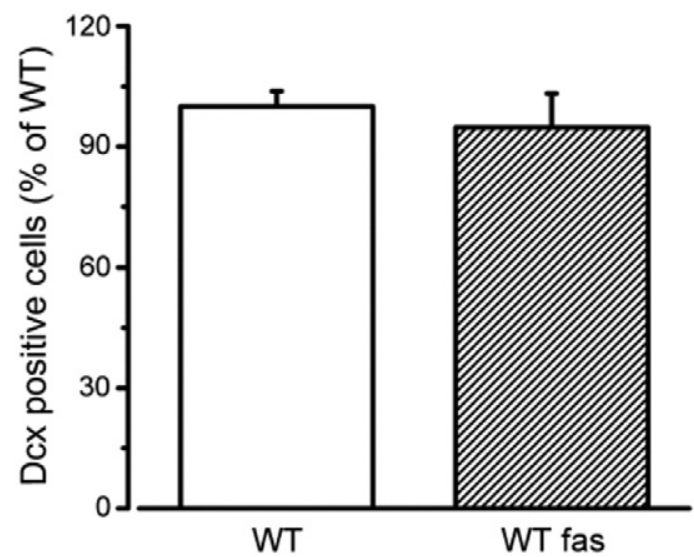

B

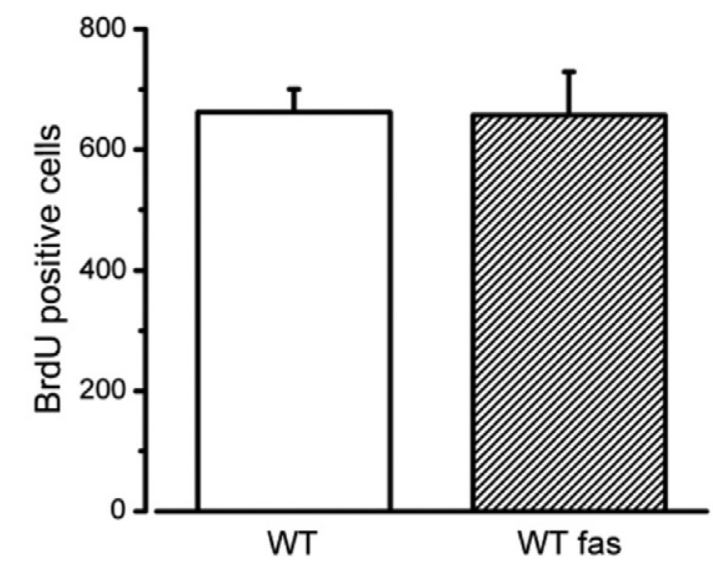

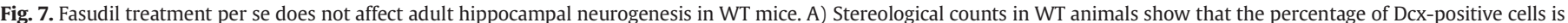

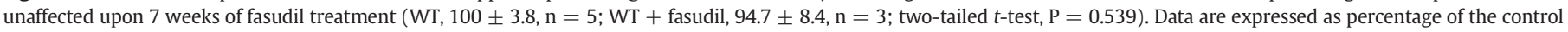

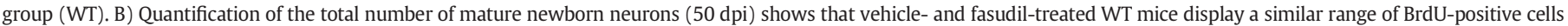
(WT, $662 \pm 38, \mathrm{n}=11$; WT + fasudil, $657 \pm 72, \mathrm{n}=4$; two-tailed $t$-test, $\mathrm{P}=0.946$ ). Histograms represent mean $\pm \mathrm{SEM}$.

activation of RhoGTPases and consequent hyperstimulation of the RhoA/ROCK pathway (Govek et al., 2004; Khelfaoui et al., 2009). In addition, lack of OPHN1 leads to high levels of PKA activity (Khelfaoui et al., 2014). Presynaptic PKA-dependent LTP, and hippocampus- and amygdala-related learning (in the fear conditioning test) are compromised in Ophn1 KO animals. Some of the electrophysiological and behavioral deficits of Ophn1 KO mice can be rescued by treatment with ROCK/PKA inhibitor fasudil (Khelfaoui et al., 2014; Meziane et al., 2016), a drug already approved for clinical use in Japan and China. Based on these premises, we tested whether chronic fasudil treatment is effective in restoring adult hippocampal neurogenesis. We found that fasudil normalized the number of Dcx-positive early neuronal cells, indicating that pharmacological ROCK/PKA inhibition may overcome the deficits caused by Ophn1 mutation at an early stage of neuronal maturation. At the morphological level, fasudil corrected the dendritic spine deficit at $21 \mathrm{dpi}$. In contrast to the dendritic effects, fasudil administration was unable to restore a normal proportion of newborn neurons extending their axon towards CA3.

Altogether, the morphological analysis reveals two key processes impacted by Ophn 1 deficiency: axonal extension and dendritic spine morphogenesis, both of which are critical for proper integration of newborn neurons (i.e. for establishing afferent and efferent connectivity). The fasudil rescue experiments indicate that these two processes proceed via at least partly independent pathways: dendritic spine density can be restored by downregulating abnormally high ROCK/PKA activity in Ophn1 KO mice, while the same approach is not effective on aberrant axonogenesis. Previous studies have shown that Ophn 1 can act as a GAP for all three Rho GTPases: RhoA, Rac1 and Cdc42 (Govek et al., 2004). Thus, the unchecked activation of all these signaling cascades may potentially explain the pathological consequences of OPHN1 deficiency. Since the expression of constitutively active Rac1 in mouse Purkinje neurons inhibits axonal outgrowth (Luo et al., 1996), an excessive Rac1-mediated signaling (not counteracted by fasudil treatment, that targets only the pathway downstream of RhoA) may explain the persistence of impaired axon formation/extension in Ophn1-deficient newborn neurons treated with fasudil.

Importantly, we found the total number of mature dentate granule neurons at 50 dpi was significantly increased by fasudil administration in Ophn1-deficient mice. Still, we observed a 20\% reduction in the number of mature neurons as compared to WT, healthy mice (see Fig. 6E).
These data indicate that only a proportion of the newly generated cells can be rescued by fasudil administration, and this is in line with the persistence of axonless newborn cells in fasudil-treated KO mice (see Fig. 6B). Obviously, adult-generated neurons unable to connect with their natural targets in the CA3 region would be eliminated from the network (despite the rescue of the dendritic spine phenotype), resulting in lower numbers of mature neurons at $50 \mathrm{dpi}$.

Overall, the present findings allow a precise dissection of alterations of neuronal maturation in XLID, and suggest a line along which possible therapeutic strategies for XLID may be developed. In particular, early fasudil treatment (i.e. in embryonic or early postnatal period) might be envisaged to correct neuronal maturation and prevent wiring deficits and consequent network dysfunction in Ophn1-dependent XLID. Given the complex pathology associated with XLID, it is likely that a combination of drugs aimed at downregulating Rho GTPase activity and administered during specific "critical periods" of brain development may be needed to successfully treat this pathology.

Supplementary data to this article can be found online at http://dx. doi.org/10.1016/j.nbd.2017.01.003.

\section{Competing interests}

The authors declare no competing interests.

\section{Authors' contributions}

Conceptualization: M.A., C.S., B.V., M. Can., M. Cal.; Formal analysis and investigation: M.A., C.S., B.V., S.A., I.B.; Writing - original draft preparation: M.A., M. Cal.; Writing - review and editing: all authors; Funding acquisition: M. Can., M. Cal.; Resources: P.B.

\section{Funding}

This work was supported by Telethon Foundation (project \#GGP11116) and Italian Ministry of Research (PRIN grant 2012MKKTNW_002 to Matteo Caleo and PRIN grant 2010N8PBAA_005 to Marco Canossa). M.A. was supported by a fellowship from Accademia dei Lince, Rome (Italy). 


\section{Acknowledgements}

\section{We thank Francesca Biondi for excellent animal care.}

\section{References}

Akers, K.G., Martinez-Canabal, A., Restivo, L., Yiu, A.P., De Cristofaro, A., Hsiang, H.L., Wheeler, A.L., Guskjolen, A., Niibori, Y., Shoji, H., Ohira, K., Richards, B.A., Miyakawa, T., Josselyn, S.A., Frankland, P.W., 2014. Hippocampal neurogenesis regulates forgetting during adulthood and infancy. Science 344, 598-602.

Bergami, M., Rimondini, R., Santi, S., Blum, R., Gotz, M., Canossa, M., 2008. Deletion of Trkb in adult progenitors alters newborn neuron integration into hippocampal circuits and increases anxiety-like behavior. Proc. Natl. Acad. Sci. U. S. A. 105, 15570-15575.

Bergami, M., Vignoli, B., Motori, E., Pifferi, S., Zuccaro, E., Menini, A., Canossa, M., 2013. Trkb signaling directs the incorporation of newly generated periglomerular cells in the adult olfactory bulb. J. Neurosci. 33, 11464-11478.

Bergami, M., Masserdotti, G., Temprana, S.G., Motori, E., Eriksson, T.M., Gobel, J., Yang, S.M., Conzelmann, K.K., Schinder, A.F., Gotz, M., Berninger, B., 2015. A critical period for experience-dependent remodeling of adult-born neuron connectivity. Neuron $85,710-717$

Billuart, P., Bienvenu, T., Ronce, N., Des Portes, V., Vinet, M.C., Zemni, R., Carrie, A., Beldjord, C., Kahn, A., Moraine, C., Chelly, J., 1998. Oligophrenin 1 encodes a rhogap protein involved in X-linked mental retardation. Pathol. Biol. (Paris) 46, 678.

Braun, S.M., Jessberger, S., 2014. Adult neurogenesis: mechanisms and functional significance. Development 141, 1983-1986.

Chelly, J., Khelfaoui, M., Francis, F., Cherif, B., Bienvenu, T., 2006. Genetics and pathophysiology of mental retardation. Eur. J. Hum. Genet. 14, 701-713.

Christian, K.M., Song, H., Ming, G.L., 2014. Functions and dysfunctions of adult hippocampal neurogenesis. Annu. Rev. Neurosci. 37, 243-262.

Christie, B.R., Cameron, H.A., 2006. Neurogenesis in the adult hippocampus. Hippocampus $16,199-207$

Contestabile, A., Greco, B., Ghezzi, D., Tucci, V., Benfenati, F., Gasparini, L., 2013. Lithium rescues synaptic plasticity and memory in down syndrome mice. J. Clin. Invest. 123 , 348-361.

Deng, W., Aimone, J.B., Gage, F.H., 2010. New neurons and new memories: how does adult hippocampal neurogenesis affect learning and memory? Nat. Rev. Neurosci. 11, 339-350.

Fauchereau, F., Herbrand, U., Chafey, P., Eberth, A., Koulakoff, A., Vinet, M.C., Ahmadian, M.R., Chelly, J., Billuart, P., 2003. The Rhogap activity of Ophn1, a new F-actin-binding protein, is negatively controlled by its amino-terminal domain. Mol. Cell. Neurosci. 23, 574-586.

Ge, S., Yang, C.H., Hsu, K.S., Ming, G.L., Song, H., 2007. A critical period for enhanced synaptic plasticity in newly generated neurons of the adult brain. Neuron 54, 559-566.

Ge, S., Sailor, K.A., Ming, G.L., Song, H., 2008. Synaptic integration and plasticity of new neurons in the adult hippocampus. J. Physiol. 586, 3759-3765.

Giacomini, A., Stagni, F., Trazzi, S., Guidi, S., Emili, M., Brigham, E., Ciani, E., Bartesaghi, R. 2015. Inhibition of app gamma-secretase restores sonic hedgehog signaling and neurogenesis in the Ts65dn mouse model of down syndrome. Neurobiol. Dis. 82, 385-396.

Govek, E.E., Newey, S.E., Akerman, C.J., Cross, J.R., Van Der Veken, L., Van Aelst, L., 2004. The X-linked mental retardation protein oligophrenin-1 is required for dendritic spine morphogenesis. Nat. Neurosci. 7, 364-372.

Guo, W., Allan, A.M., Zong, R., Zhang, L., Johnson, E.B., Schaller, E.G., Murthy, A.C., Goggin, S.L., Eisch, A.J., Oostra, B.A., Nelson, D.L., Jin, P., Zhao, X., 2011. Ablation of Fmrp in adult neural stem cells disrupts hippocampus-dependent learning. Nat. Med. 17, 559-565.

Humeau, Y., Gambino, F., Chelly, J., Vitale, N., 2009. X-linked mental retardation: focus on synaptic function and plasticity. J. Neurochem. 109, 1-14.

Iggena, D., Klein, C., Garthe, A., Winter, Y., Kempermann, G., Steiner, B., 2015. Only watching others making their experiences is insufficient to enhance adult neurogenesis and water maze performance in mice. Sci. Report. 5, 14141.

Ishihara, K., Amano, K., Takaki, E., Shimohata, A., Sago, H., Epstein, C.J., Yamakawa, K., 2010. Enlarged brain ventricles and impaired neurogenesis in the Ts1cje and Ts2cje mouse models of down syndrome. Cereb. Cortex 20,1131-1143.

Kempermann, G., Gast, D., Kronenberg, G., Yamaguchi, M., Gage, F.H., 2003. Early determination and long-term persistence of adult-generated new neurons in the hippocampus of mice. Development 130, 391-399.

Kempermann, G., Song, H., Gage, F.H., 2015. Neurogenesis in the adult hippocampus. Cold Spring Harb. Perspect. Biol. 7, A018812.

Khelfaoui, M., Denis, C., Van Galen, E., De Bock, F., Schmitt, A., Houbron, C., Morice, E., Giros, B., Ramakers, G., Fagni, L., Chelly, J., Nosten-Bertrand, M., Billuart, P., 2007. Loss of X-linked mental retardation gene oligophrenin1 in mice impairs spatial memory and leads to ventricular enlargement and dendritic spine immaturity. J. Neurosci. 27, 9439-9450.

Khelfaoui, M., Pavlowsky, A., Powell, A.D., Valnegri, P., Cheong, K.W., Blandin, Y., Passafaro, M., Jefferys, J.G., Chelly, J., Billuart, P., 2009. Inhibition of Rhoa pathway rescues the endocytosis defects in oligophrenin1 mouse model of mental retardation. Hum. Mol. Genet. 18, 2575-2583.

Khelfaoui, M., Gambino, F., Houbaert, X., Ragazzon, B., Muller, C., Carta, M., Lanore, F., Srikumar, B.N., Gastrein, P., Lepleux, M., Zhang, C.L., Kneib, M., Poulain, B., ReibelFoisset, S., Vitale, N., Chelly, J., Billuart, P., Luthi, A., Humeau, Y., 2014. Lack of the presynaptic Rhogap protein oligophrenin1 leads to cognitive disabilities through dysregulation of the camp/Pka signalling pathway. Philos. Trans. R. Soc. Lond. Ser. B Biol. Sci. 369, 20130160.

Luo, L., Hensch, T.K., Ackerman, L., Barbel, S., Jan, L.Y., Jan, Y.N., 1996. Differential effects of the Rac Gtpase on purkinje cell axons and dendritic trunks and spines. Nature 379 837-840.

Meziane, H., Khelfaoui, M., Morello, N., Hiba, B., Calcagno, E., Reibel-Foisset, S., Selloum, M., Chelly, J., Humeau, Y., Riet, F., Zanni, G., Herault, Y., Bienvenu, T., Giustetto, M. Billuart, P., 2016. Fasudil treatment in adult reverses behavioural changes and brain ventricular enlargement in oligophrenin-1 mouse model of intellectual disability. Hum. Mol. Genet.

Nadif Kasri, N., Nakano-Kobayashi, A., Malinow, R., Li, B., Van Aelst, L., 2009. The rholinked mental retardation protein oligophrenin-1 controls synapse maturation and plasticity by stabilizing ampa receptors. Genes Dev. 23, 1289-1302.

Nakano-Kobayashi, A., Kasri, N.N., Newey, S.E., Van Aelst, L., 2009. The rho-linked menta retardation protein ophn1 controls synaptic vesicle endocytosis via endophilin A1. Curr. Biol. 19, 1133-1139.

Nakano-Kobayashi, A., Tai, Y.L., Kasri, N.N., Van Aelst, L., 2014. The X-linked mental retardation protein ophn1 interacts with Homer1b/C to control spine endocytic zone positioning and expression of synaptic potentiation. J. Neurosci. 34, 8665-8671.

Plitt, M., Barnes, K.A., Wallace, G.L., Kenworthy, L., Martin, A., 2015. Resting-state functional connectivity predicts longitudinal change in autistic traits and adaptive functioning in autism. Proc. Natl. Acad. Sci. U. S. A. 112, E6699-E6706.

Pons-Espinal, M., De Lagran, M.M., Dierssen, M., 2013a. Functional implications of hippocampal adult neurogenesis in intellectual disabilities. Amino Acids 45, 113-131.

Pons-Espinal, M., Martinez De Lagran, M., Dierssen, M., 2013b. Environmental enrichment rescues Dyrk1a activity and hippocampal adult neurogenesis in Tgdyrk1a. Neurobiol. Dis. $60,18-31$

Powell, A.D., Gill, K.K., Saintot, P.P., Jiruska, P., Chelly, J., Billuart, P., Jefferys, J.G.R., 2012. Rapid reversal of impaired inhibitory and excitatory transmission but not spine dysgenesis in a mouse model of mental retardation. J. Physiol. 590 (London).

Powell, A.D., Saintot, P.P., Gill, K.K., Bharathan, A., Buck, S.C., Morris, G., Jiruska, P., Jefferys, J.G.R., 2014. Reduced gamma oscillations in a mouse model of intellectual disability: a role for impaired repetitive neurotransmission? Plos One 9

Redolfi, N., Galla, L., Maset, A., Murru, L., Savoia, E., Zamparo, I., Gritti, A., Billuart, P. Passafaro, M., Lodovichi, C., 2016. Oligophrenin-1 regulates number, morphology and synaptic properties of adult-born inhibitory interneurons in the olfactory bulb. Hum. Mol Genet.

Ropers, H.H., Hamel, B.C., 2005. X-linked mental retardation. Nat. Rev. Genet. 6, 46-57.

Rossi, C., Angelucci, A., Costantin, L., Braschi, C., Mazzantini, M., Babbini, F., Fabbri, M.E. Tessarollo, L., Maffei, L., Berardi, N., Caleo, M., 2006. Brain-derived neurotrophic factor (Bdnf) is required for the enhancement of hippocampal neurogenesis following environmental enrichment. Eur. J. Neurosci. 24, 1850-1856.

Saxe, M.D., Battaglia, F., Wang, J.W., Malleret, G., David, D.J., Monckton, J.E., Garcia, A.D., Sofroniew, M.V., Kandel, E.R., Santarelli, L., Hen, R., Drew, M.R., 2006. Ablation of hippocampal neurogenesis impairs contextual fear conditioning and synaptic plasticity in the dentate gyrus. Proc. Natl. Acad. Sci. U. S. A. 103, 17501-17506.

Schmidt-Hieber, C., Jonas, P., Bischofberger, J., 2004. Enhanced synaptic plasticity in newly generated granule cells of the adult hippocampus. Nature 429, 184-187.

Toni, N., Schinder, A.F., 2016. Maturation and functional integration of new granule cells into the adult hippocampus. Cold Spring Harb. Perspect. Biol. 8, A018903.

Valnegri, P., Khelfaoui, M., Dorseuil, O., Bassani, S., Lagneaux, C., Gianfelice, A., Benfante, R. Chelly, J., Billuart, P., Sala, C., Passafaro, M., 2011. A circadian clock in hippocampus is regulated by interaction between oligophrenin-1 and rev-Erbalpha. Nat. Neurosci. 14 1293-1301.

Van Bokhoven, H., 2011. Genetic and epigenetic networks in intellectual disabilities. Annu. Rev. Genet. 45, 81-104.

Van Der Molen, M.J., Stam, C.J., Van Der Molen, M.W., 2014. Resting-state Eeg oscillatory dynamics in fragile X syndrome: abnormal functional connectivity and brain network organization. PLoS One 9, E88451.

Vannini, E., Restani, L., Pietrasanta, M., Panarese, A., Mazzoni, A., Rossetto, O., Middei, S. Micera, S., Caleo, M., 2016. Altered sensory processing and dendritic remodeling in hyperexcitable visual cortical networks. Brain Struct. Funct. 221, 2919-2936.

Zhao, C., Teng, E.M., Summers Jr., R.G., Ming, G.L., Gage, F.H., 2006. Distinct morphological stages of dentate granule neuron maturation in the adult mouse hippocampus. J. Neurosci. 26, 3-11.

Zuccaro, E., Bergami, M., Vignoli, B., Bony, G., Pierchala, B.A., Santi, S., Cancedda, L. Canossa, M., 2014. Polarized expression of P75(Ntr) specifies axons during development and adult neurogenesis. Cell Rep. 7,138-152. 